\title{
AMICABLE ORTHOGONAL DESIGNS OF ORDER EIGHT
}

\author{
DEBORAH J. STREET
}

(Received 6 March 1981)

Communicated by W. D. Wallis

\begin{abstract}
New amicable orthogonal designs of order eight are given and they are used to construct new orthogonal designs of order 32 .
\end{abstract}

1980 Mathematics subject classification (Amer. Math. Soc.): 05 B 30.

An orthogonal design of order $n$ and type $\left(s_{1}, \ldots, s_{t}\right), s_{j} \geqslant 0$, on the commuting variables $x_{1}, x_{2}, \ldots, x_{t}$ is an $n \times n$ matrix $X$ with entries from $\left\{0, \pm x_{1}, \ldots, \pm x_{t}\right\}$ such that

$$
X X^{T}=\left(\sum_{i=1}^{t} s_{i} x_{i}^{2}\right) I_{n}
$$

Let $X$ and $Y$ be orthogonal designs of the same order $n$, where $X$ is of type $\left(s_{1}, \ldots, s_{t}\right)$ and $Y$ is of type $\left(u_{1}, \ldots, u_{v}\right)$. Then $X$ and $Y$ are amicable orthogonal designs of type $\left(\left(s_{1}, \ldots, s_{t}\right) ;\left(u_{1}, \ldots, u_{v}\right)\right)$ if $X Y^{T}=Y X^{T}$.

Amicable orthogonal designs are a useful tool in the construction of orthogonal designs. In this paper we give a summary of the known amicable orthogonal designs of order $\mathbf{8}$, including a new 'doubling' construction for such pairs. We use a design constructed in this way to give 27 new 10 variable orthogonal designs of order 32 , and to give a large number of new 6 variable designs.

We use $\bar{x}$ for $-x$ and - for -1 throughout.

C. Copyright Australian Mathematical Society 1982 
Lemma 1. Suppose $A, B, C, D$ are orthogonal designs of order $n$ such that $(A, B)$ and $(C, D)$ are both amicable pairs of type $\left(\left(a_{1}, \ldots, a_{s}\right) ;\left(b_{1}, \ldots, b_{t}\right)\right)$. Suppose further that there exists a weighing matrix $W(n, k)=W$ such that

$$
A W^{T}=W C^{T}, \quad B W^{T}=-W D^{T} .
$$

Then there exists an amicable pair of order $2 n$ of type $\left(\left(k, a_{1}, \ldots, a_{s}\right) ;\left(k, b_{1}, \ldots, b_{t}\right)\right)$.

Proof. The required designs are $\left[\frac{A}{x} W C^{x W}\right]$ and $\left[\begin{array}{ll}B W & \not b W\end{array}\right]$.

COROLlary 2. The existence of an amicable pair of type $\left(\left(a_{1}, \ldots, a_{s}\right) ;\left(b_{1}, \ldots, b_{t}\right)\right)$ in order $n$ implies the existence of an amicable pair of type $\left(\left(1, a_{1}, \ldots, a_{s}\right)\right.$; $\left.\left(1, b_{1}, \ldots, b_{t}\right)\right)$ of order $2 n$.

Proof. Let $W=I$ in the lemma.

One obvious weighing matrix to use for $W$ is a member of the Hurwitz-Radon family for $B$. However this only gives examples of pairs which can be constructed using a result of Wolfe [4]. A weaker version of that result appears in Geramita and Seberry [1, Theorem 5.48].

EXAMPLE 3. The matrices

$$
\begin{aligned}
& A=\left[\begin{array}{llll}
a & 0 & d & c \\
0 & a & \bar{c} & d \\
\bar{d} & c & a & 0 \\
\bar{c} & \bar{d} & 0 & a
\end{array}\right], \quad B=\left[\begin{array}{llll}
w & 0 & y & z \\
0 & w & z & \bar{y} \\
y & z & \bar{w} & 0 \\
z & \bar{y} & 0 & \bar{w}
\end{array}\right], \quad C=\left[\begin{array}{llll}
a & c & d & 0 \\
\bar{c} & a & 0 & d \\
\bar{d} & 0 & a & \bar{c} \\
0 & \bar{d} & c & a
\end{array}\right], \\
& D=\left[\begin{array}{llll}
\bar{z} & \bar{y} & \bar{w} & 0 \\
\bar{y} & z & 0 & \bar{w} \\
\bar{w} & 0 & z & y \\
0 & \bar{w} & y & \bar{z}
\end{array}\right] \quad \text { and } \quad W=\left[\begin{array}{cccc}
1 & 1 & 1 & 1 \\
1 & - & 1 & - \\
1 & 1 & - & - \\
1 & - & - & 1
\end{array}\right]
\end{aligned}
$$

satisfy the conditions of Lemma 1 and give an amicable pair of type $((1,1,1,4)$; $(1,1,1,4))$ of order 8 . This pair is new.

Using designs constructed from Wolfe's result, the $((1,1,1,4) ;(1,1,1,4))$ from above and [1, Theorem 5.97] gives the following 27 10-tuples which are the types of new 10 variable orthogonal designs in order 32; see Seberry [3] for the current status of the existence of 10 variable orthogonal designs of order 32 . The 
10-tuples are

$\begin{array}{ll}(1,1,1,1,1,1,1,3,3,3), & (1,1,1,2,2,2,4,4,4,4), \\ (1,1,1,1,1,1,2,2,2,4), & (1,1,1,2,2,2,4,5,5,5), \\ (1,1,1,1,1,1,2,2,4,8), & (1,1,1,3,3,3,3,3,3,3), \\ (1,1,1,1,1,1,2,3,3,6), & (1,1,1,3,3,3,4,4,4,4), \\ (1,1,1,1,1,1,2,4,4,4), & (1,1,2,2,2,2,2,2,2,2), \\ (1,1,1,1,1,1,3,3,4,12), & (1,1,2,2,2,2,2,3,3,3), \\ (1,1,1,1,1,1,4,4,4,4), & (1,1,2,2,2,2,2,3,3,6), \\ (1,1,1,1,1,1,4,5,5,5), & (1,1,2,2,2,2,2,4,4,4), \\ (1,1,1,1,1,1,4,6,6,6), & (1,1,2,2,3,3,3,3,3,3), \\ (1,1,1,1,1,2,2,2,4,4), & (1,1,2,3,3,3,3,3,3,3), \\ (1,1,1,1,1,2,2,4,4,4), & (1,1,2,3,3,3,3,4,4,4), \\ (1,1,1,1,1,2,2,5,5,5), & (2,2,2,2,2,2,2,2,2,2), \\ (1,1,1,1,1,2,3,6,6,6), & (2,2,2,2,2,2,2,4,4,4), \\ (1,1,1,2,2,2,2,3,3,3), & \end{array}$

Using these designs, those in [3], and results listed in Appendix $G$ of [1] we have the following result.

LEMMA 4. In order 32 all 6-tuples of weight less than or equal to 28 are the types of orthogonal designs of order 32 except possibly

$$
\begin{array}{ll}
(2,2,2,3,7,9), & (1,1,1,5,8,11), \\
(1,1,1,1,1,21), & (1,2,2,2,3,17), \\
(1,1,1,2,5,17), & (1,2,2,3,8,11), \\
(1,1,1,5,5,14) . &
\end{array}
$$

Finally we give tables which summarize the known results about the existence and non-existence of amicable pairs of order 8 . In these tables

$A$ means that such a pair can not exist by virtue of

$B$ means that such a pair can not exist by virtue of

$C$ means that such a pair can not exist by virtue of

$D$ means that such a pair can not exist by virtue of

$F$ means that such a pair can not exist by virtue of

$G$ means that such a pair can not exist by virtue of

48 means that such a pair can be constructed using Wolfe's construction,

52 means that such a pair can be constructed using

58 means that such a pair can be constructed using

64 means that such a pair can be constructed using

95 means that such a pair can be constructed using
[1, Theorem 5.39],

[1, Theorem 5.41],

[1, Theorem 5.45],

[1, Theorem 5.47],

[1, p. 240],

[1, Theorem 5.64],

[1, Theorem 5.52],

[1, Theorem 5.58],

[1, Theorem 5.64],

[1, Theorem 5.95], 
6 means that such a pair can be constructed using [1, Table 5.6],

[2] means that such a pair is given in [2], and

* means that such a pair can be constructed using Example 3.

The number of variables in each member of a pair is shown in the caption of the table.

\section{Table 1}

Both designs with 4 variables

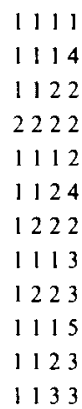

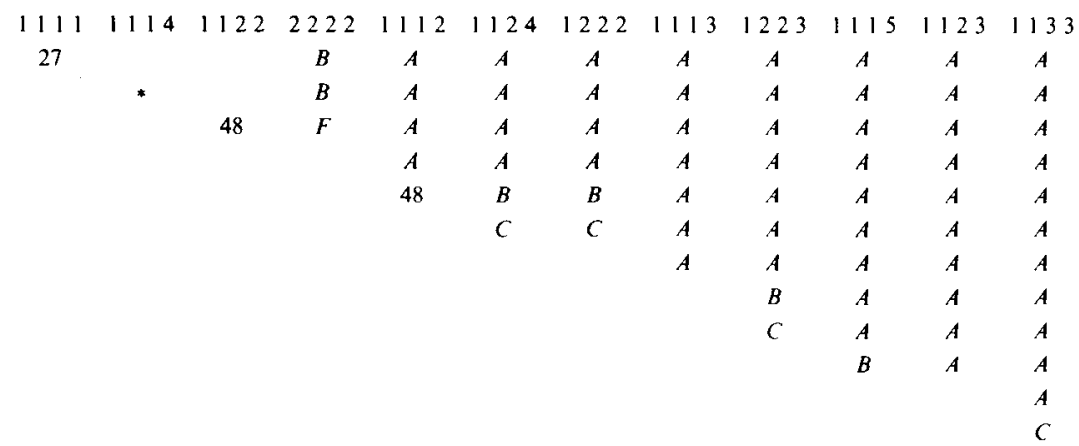

Table 2

Designs with 4 and 3 variables

\begin{tabular}{|c|c|c|c|c|c|c|c|c|c|c|c|c|}
\hline & 1111 & 1114 & 1122 & 2222 & 1112 & 1124 & 1222 & 1113 & 1223 & 1123 & 1115 & 1133 \\
\hline 111 & 48 & * & & $B$ & 95 & $B$ & & & $B$ & $A$ & $B$ & $A$ \\
\hline 114 & & * & & $F$ & & $C$ & & & $C$ & $A$ & $C$ & $A$ \\
\hline 122 & & & 95 & $F$ & 95 & $C$ & & & $C$ & $A$ & $C$ & $A$ \\
\hline 224 & $B$ & $B$ & & 48 & $B$ & 48 & & $B$ & $F$ & $A$ & $B$ & $A$ \\
\hline 112 & 95 & & 48 & $F$ & 48 & $C$ & & $A$ & $A$ & & $A$ & $A$ \\
\hline 124 & & * & & $F$ & & $C$ & & $A$ & $A$ & & $A$ & $A$ \\
\hline 222 & & & 48 & & & & & $A$ & $A$ & & $A$ & $A$ \\
\hline 113 & $A$ & $A$ & A & $A$ & 6 & $C$ & & & $C$ & & $A$ & $C$ \\
\hline 134 & $A$ & $A$ & $A$ & $A$ & $B$ & C & $C$ & $B$ & $C$ & C & $A$ & $C$ \\
\hline 223 & $A$ & $A$ & $A$ & $A$ & $B$ & $F$ & & $B$ & $F$ & & $A$ & $F$ \\
\hline 123 & & & 48 & $F$ & $A$ & $A$ & $A$ & & $C$ & & $A$ & $A$ \\
\hline 115 & & * & & $D$ & $A$ & $A$ & $A$ & $A$ & & & $C$ & $A$ \\
\hline 133 & $A$ & $A$ & $A$ & $A$ & $A$ & $A$ & $A$ & $A$ & $A$ & $\sigma$ & $A$ & $C$ \\
\hline 116 & $A$ & $A$ & $A$ & $A$ & $B$ & $C$ & $C$ & $A$ & $A$ & $A$ & $B$ & $C$ \\
\hline 125 & $A$ & $A$ & $A$ & $A$ & $B$ & $C$ & $C$ & $B$ & $C$ & $A$ & $B$ & $A$ \\
\hline 233 & $A$ & $A$ & $A$ & $A$ & $A$ & $A$ & $A$ & $B$ & $F$ & $A$ & $A$ & $D$ \\
\hline
\end{tabular}


Table 3

Designs with 4 and 2 variables

$\begin{array}{lccccccccccccc}11 & 1111 & 1114 & 1122 & 2222 & 1112 & 1124 & 1222 & 1113 & 1223 & 1123 & 1115 & 1133 \\ 14 & & * & 48 & F & 48 & C & & & C & & C & C \\ 22 & 6 & & 45 & F & 6 & C & & & C & & C & C \\ 44 & B & B & & 48 & B & 48 & & B & F & D & B & D \\ 12 & 48 & * & 48 & F & 48 & C & & & C & & C & A \\ 24 & & * & 48 & 48 & & 48 & & & & & & A \\ 13 & 95 & & 48 & F & 48 & C & & & C & 6 & A & C \\ 34 & & * & & & & & & & & 6 & A & \\ 15 & & * & 48 & F & & C & & & C & & C & A \\ 15 & & * & & F & & C & & A & A & 6 & C & C \\ 17 & A & A & A & A & B & C & F & B & C & D & B & C \\ 23 & & & 95 & & 6 & F & & & F & & F & F \\ 25 & & * & & & & F & & & F & & F & F \\ 26 & B & B & & 48 & B & 48 & & B & 64 & A & B & D \\ 33 & & & 48 & & A & A & A & 48 & & 48 & A & \\ 35 & B & B & & & B & F & & B & F & D & B & D\end{array}$

Table 4

Designs with 4 and 1 variables

$\begin{array}{cccccccccccccc} & 1111 & 1114 & 1122 & 2222 & 1112 & 1124 & 1222 & 1113 & 1223 & 1115 & 1123 & 1133 \\ 1 & 48 & * & 48 & F & 48 & C & & & C & C & 6 & C \\ 2 & 48 & * & 48 & 48 & 48 & 48 & & & 64 & & & \\ 3 & 48 & * & 48 & & 48 & & & 48 & & & 48 \\ 4 & 6 & * & 48 & 48 & 48 & 48 & & & & & & \\ 5 & & * & 95 & & 6 & F & & & F & F & & F \\ 6 & & * & 48 & 48 & & 48 & 48 & 64 & & 48 & \\ 7 & & * & & & & & & & & 6 & \\ 8 & B & B & & 48 & B & 48 & & B & 64 & B & D & D\end{array}$

Table 5

Both designs with 3 variables

$111114 \quad 122224 \quad 112124222 \quad 113223 \quad 134 \quad 123 \quad 115 \quad 133 \quad 116 \quad 125 \quad 233$ 
Table 6

Designs with 3 and 2 variables

$\begin{array}{ccccccccccccccccc}11 & 111 & 114 & 122 & 224 & 112 & 124 & 222 & 113 & 223 & 134 & 123 & 115 & 133 & 116 & 125 & 233 \\ 14 & 58 & * & 95 & 48 & 48 & * & 48 & 6 & & C & 95 & * & 6 & C & C & \\ 22 & 48 & 48 & 48 & 48 & 48 & 48 & 48 & 48 & & 48 & 48 & & 48 & 48 & 48 & 48 \\ 44 & B & 48 & & 48 & 48 & 48 & 48 & D & & 48 & & D & & 48 & 48 & 48 \\ 12 & 48 & * & 48 & 48 & 48 & * & 48 & 48 & & C & 48 & * & 48 & C & C & \\ 24 & * & 48 & 48 & 48 & 48 & 48 & 48 & & & 48 & 48 & * & 6 & 48 & 48 & 48 \\ 13 & 48 & 48 & 48 & 48 & 48 & 6 & 48 & 48 & 6 & C & 48 & 6 & 48 & C & C & \\ 34 & * & * & & 48 & 6 & 6 & & 6 & 6 & & 6 & 6 & 58 & & & * \\ 15 & * & * & 95 & 48 & 48 & * & 48 & & & C & 48 & * & 6 & C & C & \\ 16 & * & * & & 48 & 6 & 6 & & 6 & 6 & C & 6 & 6 & 6 & C & C & \\ 17 & B & F & F & 48 & & F & & D & F & C & F & D & & C & C & F \\ 23 & 58 & 48 & 58 & & 48 & & 48 & 58 & & F & 95 & & 58 & F & F \\ 25 & * & * & & 48 & & * & & & & F & & * & 6 & F & F \\ 26 & B & 48 & 64 & 48 & 48 & 48 & 48 & D & 64 & 48 & 64 & D & & 48 & 48 & 48 \\ 33 & 48 & & 95 & 48 & 48 & 6 & 48 & 48 & 48 & & 48 & 6 & 48 & & F \\ 35 & B & & & 48 & & & & D & & F & & D & & F & F\end{array}$

Table 7

Designs with 3 and 1 variables

$\begin{array}{ccccccccccccccccccc} & 111 & 114 & 122 & 224 & 112 & 124 & 222 & 113 & 223 & 134 & 123 & 115 & 133 & 116 & 125 & 233 \\ 1 & 48 & * & 48 & 48 & 48 & 6 & 48 & 48 & 6 & C & 48 & 6 & 48 & C & C & \\ 2 & 48 & 48 & 48 & 48 & 48 & 48 & 48 & 48 & 64 & 48 & 48 & * & 48 & 48 & 48 & 48 \\ 3 & 48 & * & 48 & 48 & 48 & 6 & 48 & 48 & 48 & & 48 & 6 & 48 & \\ 4 & 48 & 48 & 48 & 48 & 48 & 48 & 48 & 48 & 95 & 48 & 48 & * & 48 & 48 & 48 & 48 \\ 5 & 58 & * & 58 & 48 & 48 & * & 48 & 6 & & F & 95 & * & 58 & F & F & 48 \\ 6 & 48 & 48 & 48 & 48 & 48 & 48 & 48 & 48 & 48 & 48 & 48 & 6 & 48 & 48 & 48 & 48 \\ 7 & * & * & & 48 & 6 & 6 & & 6 & 6 & & 6 & 6 & 58 & & & & & \\ 8 & B & 48 & 64 & 48 & 48 & 48 & 48 & D & 64 & 48 & 64 & D & & 48 & 48 & 48\end{array}$

Table 8

Both designs with 2 variables

\begin{tabular}{|c|c|c|c|c|c|c|c|c|c|c|c|c|c|c|c|}
\hline & 11 & 12 & 13 & 14 & 15 & 16 & 17 & 22 & 23 & 24 & 25 & 26 & 33 & 34 & 35 \\
\hline 11 & $4 x$ & 48 & 48 & 48 & 48 & 48 & & 48 & 48 & 48 & * & 48 & 48 & 48 & \\
\hline 12 & & 48 & 48 & 48 & 48 & 48 & & $4 X$ & 48 & 48 & $*$ & 48 & 48 & 48 & \\
\hline 13 & & & $4 x$ & 48 & 48 & 48 & & 48 & 48 & 48 & 6 & 48 & 48 & 48 & \\
\hline 14 & & & & 6 & 95 & 6 & $F$ & 48 & 58 & 48 & * & 48 & $4 x$ & 6 & \\
\hline 15 & & & & & $*$ & 6 & $F$ & $4 X$ & 95 & 48 & * & 48 & 48 & 6 & \\
\hline 16 & & & & & & 6 & & 48 & 58 & 48 & 6 & 48 & $4 x$ & 58 & \\
\hline 17 & & & & & & & 52 & 48 & $F$ & 48 & $F$ & 48 & & & $F$ \\
\hline 22 & & & & & & & & $4 x$ & 48 & $4 k$ & 48 & 48 & 48 & 48 & 48 \\
\hline 23 & & & & & & & & & 58 & $4 x$ & & 48 & $4 x$ & 58 & \\
\hline 24 & & & & & & & & & & 48 & 48 & 48 & $4 x$ & 48 & 48 \\
\hline 25 & & & & & & & & & & & * & 48 & $4 x$ & 6 & \\
\hline 26 & & & & & & & & & & & & 48 & 48 & 48 & 48 \\
\hline 33 & & & & & & & & & & & & & 48 & 48 & \\
\hline 34 & & & & & & & & & & & & & & 58 & \\
\hline 35 & & & & & & & & & & & & & & & \\
\hline 44 & & & & & & & & & & & & & & & \\
\hline
\end{tabular}


Table 9

Designs with 2 and 1 variables

$\begin{array}{lllllllllllllllll} & 11 & 12 & 13 & 14 & 15 & 16 & 17 & 22 & 23 & 24 & 25 & 26 & 33 & 34 & 35 & 44 \\ 1 & 48 & 48 & 48 & 48 & 48 & 48 & 52 & 48 & 48 & 48 & 6 & 48 & 48 & 48 & & 48 \\ 2 & 48 & 48 & 48 & 48 & 48 & 48 & 48 & 48 & 48 & 48 & 48 & 48 & 48 & 48 & 48 & 48 \\ 3 & 48 & 48 & 48 & 48 & 48 & 48 & {[2]} & 48 & 48 & 48 & 6 & 48 & 48 & 48 & & 48 \\ 4 & 48 & 48 & 48 & 48 & 48 & 48 & 48 & 48 & 48 & 48 & 48 & 48 & 48 & 48 & 48 & 48 \\ 5 & 48 & 48 & 48 & 58 & 95 & 58 & F & 48 & 58 & 48 & * & 48 & 48 & 58 & & 48 \\ 6 & 48 & 48 & 48 & 48 & 48 & 48 & 48 & 48 & 48 & 48 & 48 & 48 & 48 & 48 & 48 & 48 \\ 7 & 48 & 48 & 48 & 6 & 6 & 58 & 52 & 48 & 58 & 48 & 6 & 48 & 48 & 58 & & 48 \\ 8 & 48 & 48 & 48 & 48 & 48 & 48 & 48 & 48 & 48 & 48 & 48 & 48 & 48 & 48 & 48 & 48\end{array}$

NOTE ADDED IN PROOF. Jennifer Seberry has recently informed me that she has constructed all the designs mentioned in Lemma 4 except the $(1,1,1,5,5,14)$ design.

ACKNOWLEDGement. I thank Dr. Jennifer Seberry for her help and encouragement.

\section{References}

[1] Anthony V. Geramita and Jennifer Seberry, Orthogonal designs: quadratic forms and Hadamard matrices (Marcel Dekker, New York, 1979).

[2] Peter J. Robinson and Jennifer Seberry, 'On the structure and existence of some amicable orthogonal designs', J. Austral. Math. Soc. 25 (1978), 118-128.

[3] Jennifer Seberry, '10-tuples in order 32 which satisfy necessary conditions' (Research Report. Department of Applied Mathematics, University of Sydney, 1980).

[4] Warren W. Wolfe, Orthogonal designs-amicable orthogonal designs-some algehraic and combinatorial techniques (Ph.D. Dissertation, Queen's University, Kingston, Ontario, 1975).

\section{Department of Mathematics}

University of Queensland

St. Lucia, Queensland 4067

Australia 